\title{
Terapia nutricional imunomoduladora para paciente oncológico no perioperatório gastrintestinal: protocolo de revisão de escopo
}

Immunomodulatory nutritional therapy for cancer patients in the gastrointestinal perioperative period: scope review protocol

Terapia nutricional inmunomoduladora para pacientes con cáncer en el período perioperatorio gastrointestinal: protocolo de revisión del alcance

Recebido: 19/11/2021 | Revisado: 28/11/2021 | Aceito: 03/12/2021 | Publicado: 19/12/2021

\author{
Heliane Formagio Silva \\ ORCID: https://orcid.org/0000-0003-1891-9763 \\ Hospital Regional de Cacoal, Brasil \\ E-mail:helianenutricao@gmail.com \\ Patrícia de Souza Chagas \\ ORCID: https://orcid.org/0000-0003-1621-5228 \\ Hospital Regional de Cacoal, Brasil \\ E-mail: patty_chagas00@ hotmail.com \\ Emanuelle Nogueira Negreiros \\ ORCID: https://orcid.org/0000-0002-0572-8231 \\ Hospital Regional de Cacoal, Brasil \\ E-mail:emanuellenutri@ hotmail.com \\ Laurindo Pereira de Souza \\ ORCID: https://orcid.org/0000-0002-9890-2621 \\ Hospital Regional de Cacoal, Brasil \\ E-mail: laurindosorrisox@hotmail.com
}

\begin{abstract}
Resumo
Objetivo: O objetivo proposto nessa pesquisa é mapear estudos clínicos que avaliam os possíveis benefícios da Terapia nutricional imunomoduladora em paciente oncológico que realizaram cirurgias gastrintestinais. Método: tratase de um protocolo de estudos do tipo scoping review proposto por Joanna Brigges Intitute. Foram revisados estudos nos idiomas: inglês e português e, a base de dados selecionada para busca foi a Literatura Latino-Americana e do Caribe em Ciências da Saúde (LILACS), MEDELINE/PUBMED e literatura cinzenta. O protocolo em estudo está registrado na plataforma Open Science Framework (OSF), sob o registro osf.io/ef7z9 e doi 10.17605/OSF.IO/YSHF3. Resultados esperados: Um mapeamento de estudos que avaliarão o desfecho clínico de pacientes oncológicos que receberam terapia nutricional imunomoduladora no perioperatório de cirurgia gastrintestinal. Conclusão: Espera-se que esta revisão de escopo possa encontrar evidências que comprovem possíveis benefícios apresentados com a terapia nutricional imunomoduladora em pacientes oncológicos.
\end{abstract}

Palavras-chave: Terapia nutricional; Oncologia cirúrgica; Neoplasias gástricas; Imunomodulação.

\begin{abstract}
Objective: the objective proposed in this research is to map clinical studies that evaluated the possible benefits of immunomodulatory nutritional Therapy in cancer patients who underwent gastrointestinal surgeries. Method: this is a study protocol of the lotecoping review proposed by Joanna Brigges Intitute. Studies were reviewed in the languages: English and Portuguese, the database selected for the search was, Latin American and Caribbean Literature in Health Sciences (LILACS), MEDELINE/PUBMED and gray literature, the study protocol is registered in the Open platform Science Framework (OSF), under registration osf.io/ef7z9 and doi 10.17605/OSF.IO/YSHF3. Expected Results: A mapping of studies that will assess the clinical outcome of cancer patients who received immunomodulatory nutritional therapy in the perioperative period of gastrointestinal surgery. Conclusion: It is expected that this scope review can find evidence that proves possible benefits presented with immunomodulatory nutritional therapy in cancer patients.
\end{abstract}

Keyword: Nutritional therapy; Surgical oncology; Gastric neoplasms; Immunomodulation.

\section{Resumen}

Objetivo: el objetivo propuesto en esta investigación es mapear estudios clínicos que evaluaran los posibles beneficios de la Terapia nutricional inmunomoduladora en pacientes oncológicos sometidos a cirugías gastrointestinales. Método: se trata de un protocolo de estudio de la revisión de lotecoping propuesto por Joanna Brigges Intitute. Los 
estudios fueron revisados en los idiomas: inglés y portugués, la base de datos seleccionada para la búsqueda fue, Literatura Latinoamericana y del Caribe en Ciencias de la Salud (LILACS), MEDELINE / PUBMED y literatura gris, el protocolo de estudio está registrado en la plataforma abierta Science Framework ( OSF), con registro osf.io/ef7z9 y doi 10.17605 / OSF.IO / YSHF3. Resultados esperados: un mapeo de estudios que evaluarán el resultado clínico de los pacientes con cáncer que recibieron terapia nutricional inmunomoduladora en el período perioperatorio de cirugía gastrointestinal. Conclusión: Se espera que esta revisión del alcance pueda encontrar evidencia que demuestre los posibles beneficios que presenta la terapia nutricional inmunomoduladora en pacientes con cáncer.

Palabra clave: Terapia nutricional; Oncología quirúrgica; Neoplasias gástricas; Inmunomodulación.

\section{Introdução}

Entre as patologias existentes, o câncer abala diretamente o perfil nutricional do indivíduo, ocasionando variações metabólicas, funcionais e morfológicas. Durante o processo de intervenção à doença, operações cirúrgicas são comumente realizadas, deste modo, conhecer e mediar no perioperatório o estado nutricional, impactará em sua recuperação. Estudos mostram que a desnutrição se apresenta recorrente em pacientes cirúrgicos, e destes, 35\% a $60 \%$ são pacientes oncológicos (Sierra et al., 2020). A sarcopenia também é uma das complicações causadas pelo câncer. Seu diagnóstico e tratamento é de extrema importância para o desfecho clínico do paciente. A diminuição da massa magra impacta em aumento da mortalidade, e assim como a desnutrição, está relacionada ao déficit nutricional. Dar atenção a perda de massa magra é uma importante estratégia para mitigar as complicações e seus impactos na saúde pública (Au et al., 2021).

A desnutrição proteico-energética é comumente identificada entre os pacientes oncológicos, e está relacionada com a baixa ingestão de macro e micronutrientes (Tonon et al., 2020). A recusa para com o alimento é caracterizada por alterações como mucosite, xerostomia e a disfagia, e o tratamento para neoplasias realizado através da quimioterapia e radioterapia, tendem a aumentar os efeitos colaterais, agravando ainda mais o estado nutricional do paciente oncológico (Tiezerin et al., 2021).

O cuidado nutricional inadequado e a ausência de monitoramento frequente do estado nutricional desses pacientes, estão entre os riscos que podem causar a desnutrição. O nutricionista deve realizar uma boa avaliação do estado nutricional e das necessidades nutricionais do paciente, além de realizar aconselhamento dietético, educação e acompanhamento do seguimento da dieta, assim como manejar possíveis intercorrências relacionadas a terapia nutricional. Com isso, a integração entre intervenção nutricional precoce e uma boa relação com o paciente, constituem a chave para o sucesso na sua recuperação (Cardoso et al., 2019).

\section{Metodologia}

Refere-se a um protocolo de estudo de conclusão de residência (TCR), vinculado ao Programa de Residência Multiprofissional em Terapia Intensiva do Hospital Regional de Cacoal, Rondônia (Brasil), que terá como objetivo mapear as evidências inerentes ao impacto da Terapia nutricional imunomoduladora em paciente oncológico submetido a cirurgia gastrintestinal. Como orientação metodológica, a pesquisa será fundamentada por Joanna Briggs Intitute (JBI). Este protocolo em estudo foi registrado na plataforma Open Science Framework (OSF), sob registroosf.io/ef7z9e doi10.17605/OSF.IO/YSHF3.

Com base nessas informações, a justificativa e a relevância desta pesquisa são de mapear evidências que apresentem os resultados encontrados na intervenção com fórmulas imunomoduladoras no perioperatório de pacientes oncológicos.

Neste contexto, realizou-se previamente uma busca nas plataformas Joana Briggs Institute (JBI), Literatura LatinoAmericana e do Caribe em Ciências da Saúde (LILACS), MEDLINE/PubMed. Entretanto, nenhum estudo do tipo revisão da literatura foi encontrado entre o recorte de setembro a outubro de 2021. Dessa forma, os pesquisadores optaram em realizar 
uma Scoping Review, norteado pela metodologia referência internacional nesse tipo de estudo, proposta por Joanna Briggs Intitute para Scoping Reviews (Aromataris et al., 2020; Jordan et al., 2019).

As revisões de escopo possuem ampla prestatividade em diversas disciplinas, entre estas podemos incluir ciências da saúde. Este estudo pode ser utilizado para atualizar os leitores nas evidências que apontam os resultados observados em pacientes oncológicos referentes ao seu estado nutricional e condutas de intervenções nutricionais que propõem mudanças no desfecho clínico no pós-operatório.

Adverso a uma revisão sistemática, as revisões de escopo visam fornecer uma visão geral do estudo proposto, através de mapeamento das evidências, podendo ser usadas para esquematizar os conceitos-chave que sustentam um campo de pesquisa, esclarecendo as definições de trabalhos. A revisão de escopo baseia-se em explorar a amplitude ou extensão da literatura, mapear e resumir as evidências e informar novas pesquisas (Peters et al., 2020).

A construção do protocolo dessa revisão de escopo segue as definições exigidas pelo check list, Preferred Reporting Items for Systematic reviews and Meta Analyses extension for Scoping Reviews (PRISMA-ScR) que se traduz em um roteiro que orienta para a escrita do relatório de revisão de escopo. O PRISMA-ScR é constituído por 22 rótulos fragmentados em seções indispensáveis para estrutura da pesquisa: Título, Resumo, Introdução, Método, Resultados, Discussão e Financiamento (Tricco et al, 2018).

\subsection{Estratégia de pesquisa}

\section{Questões de pesquisa e critérios de busca}

A pesquisa que se pretende desenvolver buscará responder a seguinte pergunta norteadora: existem estudos científicos, que versem sobre a terapia nutricional imunomoduladora apresentando benefícios no pós operatório de cirurgias gastrintestinal em pacientes oncológicos?

Para responder à pergunta norteadora será utilizado a estratégia PCC que responde a Population(População), Concept(Conceito), e Context(contexto). Nessa perspectiva, a revisão será guiada de modo a incluir estudos de intervenção que traçaram como objetivo avaliar os benefícios de formulas imunomoduladoras no perioperatorio gastrintestinal em pacientes oncológicos.

Quanto ao contexto, serão integrados todos os aspectos clínicos ou regiões geográficas nacionais e internacionais. Respeitando ao tipo de estudo, esta revisão irá considerar desenhos do tipo ensaio clínico randomizado, estudo de coorte retrospectiva. Adicionalmente, serão consideradas literatura cinzenta e opiniões de especialistas.

Com base nos critérios PCC, a estratégia de pesquisa e identificação dos estudos serão utilizadas as bases de dados, Literatura Latino-Americana e do Caribe em Ciências da saúde (LILACS), MEDELINE/PUBMED e literatura cinzenta.

Para a busca dos estudos, foram selecionadas as bases de dados PubMed e Biblioteca Virtual em Saúde (BVS). Em cada base de dados, os descritores controlados foram delimitados pelos Descritores em Ciência da Saúde (DeCS). Os descritores utilizados estão elencados em primeira ordem em inglês: 1- Nutrition Therapy; 2 - Surgical Ongology; 3 Malnutrition; 4 Stomach Neoplasms, e em português 1 - Terapia Nutricional; 2 - Oncologia cirúrgica; 3 - Desnutrição; 4 Neoplasias Gástricas.

\section{Gerenciamento de citações}

Os autores utilizaram para gestão de referências e remoção de duplicidade o Mendeley (Copyright@2009-2013 Mendeley Ltd. https://www.mendeley.com/download-desktop-new/) e os artigos foram gerenciados usando planilhas do Excel, sendo que em um segundo momento, foi feita uma reanálise de duplicidade no Excel para garantir a confiança da seleção, e 
após findar esta fase de organização dos dados, os revisores independentes se reunirão para resolver conflitos e discutir quaisquer incertezas relacionadas à seleção do estudo.

\section{Critério de elegibilidade}

$\mathrm{Na}$ busca de garantir a credibilidade na escolha das informações, foi utilizado um instrumento de coleta que descreve todas as informações pertinentes que respondem à questão norteadora do estudo (Oliveira et al., 2021).

Esta fase será conduzida por dois revisores independentes, tendo em vista aos recursos limitados para tradução, onde serão excluídos artigos em idiomas que não o português e inglês.

O instrumento de coleta de dados do estudo terá as informações apresentadas no quadro 1. Os resultados da pesquisa serão relatados na íntegra na revisão do escopo final e apresentados em um diagrama de fluxo de itens de Revisão de Escopo (PRISMA-ScR) (Tricco et al, 2018).

Quadro 1. Instrumento de coleta de informações dos estudos que serão selecionados para revisão de escopo final (Oliveira $e t$ al., 2021).

\begin{tabular}{|c|c|c|c|c|}
\hline \multicolumn{5}{|c|}{ Título do estudo } \\
\hline \multicolumn{5}{|c|}{ Terapia Nutricional imunomoduladora para paciente oncológico no perioperatório gastrintestinal: protocolo de revisão de escopo } \\
\hline \multicolumn{5}{|c|}{ Pergunta norteadora } \\
\hline \multicolumn{5}{|c|}{$\begin{array}{l}\text { Existem estudos científicos, que versem sobre a terapia nutricional imunomoduladora apresentando benefícios no pós operatório de cirurgias } \\
\text { gastrintestinal em pacientes oncológicos? }\end{array}$} \\
\hline \multicolumn{5}{|c|}{ Objetivo } \\
\hline \multicolumn{5}{|c|}{$\begin{array}{l}\text { O objetivo proposto nesta pesquisa é mapear estudos clínicos que avaliaram os possíveis benefícios da Terapia Nutricional imunomoduladora em } \\
\text { pacientes oncológicos que realizaram cirurgias gastrintestinais. }\end{array}$} \\
\hline \multicolumn{3}{|c|}{ População } & & $\mathbf{P}$ \\
\hline \multicolumn{5}{|c|}{ Pacientes oncológicos que irão realizar cirurgia gastrintestinal. } \\
\hline \multicolumn{3}{|c|}{ Conceito } & & $\mathbf{C}$ \\
\hline \multicolumn{5}{|c|}{$\begin{array}{l}\text { Estudos focados em responder a relação entre a Terapia Nutricional imunomoduladora, e desfechos clínicos relacionados a infecções, tempo de } \\
\text { internação e custos hospitalares. }\end{array}$} \\
\hline \multicolumn{3}{|c|}{ Contexto } & & $\mathbf{C}$ \\
\hline \multicolumn{5}{|c|}{ Todos os aspectos clínicos ou regiões geográficas nacional e internacional. } \\
\hline \multicolumn{5}{|c|}{ Extração de detalhes e perfil dos estudos } \\
\hline \multicolumn{5}{|c|}{ Artigos } \\
\hline Base de dados & Localizados & Excluídos & Analisados & Idiomas \\
\hline bela 2. Distr & ibuição dos estudos & autores, & po de estudo & tivo, na revisão escopo final, 2021. \\
\hline Autor & Ano de publicação & Tipo de estudo & Qualis da revista & Objetivo \\
\hline & & & & \\
\hline
\end{tabular}

Fonte: Autores.

\section{Síntese de dados e ética de pesquisa}

$\mathrm{Na}$ exposição dos resultados finais apresentados na revisão escopo, serão utilizadas tabelas e fluxogramas obedecendo a exigência estrutural do mesmo. A apresentação da síntese de dados será feita de forma narrativa, de modo que possa ser possível observar, pontuar e classificar os dados no intuito de reunir conhecimento sobre o estudo explorado nesta revisão de escopo. 
Levando em conta os aspectos éticos, nesta revisão de escopo é assegurada a autoria dos artigos pesquisados, de maneira que as fontes bibliográficas, artigos científicos e base de dados foram citados. Para este estudo foi escusado a anuência do Comitê de Ética e Pesquisa-CEP, por não serem envolvidos seres humanos neste estudo, tratando-se então de uma revisão da literatura.

\section{Resultados e Discussão}

Em uma busca realizada nas bases de dados, foram encontrados 23 estudos com potencial relação ao tema abordado. Após fazer uma leitura do título e do resumo, foram excluídos 12 destes estudos por não contribuírem para a área temática em questão. Dos 11 estudos potencialmente relevantes, foram excluídos 6 por se tratar de estudos de revisão e 1 por não ser com pacientes oncológicos. Seguindo a linha de seleção criteriosa, foram incluídos na pesquisa 4 trabalhos que se apresentaram pertinentes.

Os estudos selecionados foram estudos Clínicos randomizados, realizados entre os anos de 1994 a 2012, ocorridos no Brasil, Itália e Alemanha. Os mesmos priorizaram como conduta nutricional uma suplementação imunomoduladora no perioperatório de pacientes oncológicos.

A realização desta pesquisa foi organizada de forma criteriosa, buscando selecionar trabalhos que atendessem os mesmos métodos de forma homogênea, podendo colaborar com dados estatísticos que respondam as hipóteses levantadas sobre a temática abordada, desta forma, sendo possível reunir estudos relevantes na literatura.

Durante o processo de revisão da mesma a principal limitação encontrada foi a ausência de estudos recentes, de forma que o último foi concluído no ano de 2012 e publicado em 2013. Entretanto, os trabalhos selecionados se mostraram uniformes dentro dos critérios metodológicos estabelecidos. Ademais, os dados estatísticos encontrados se apresentaram satisfatórios para responder à pergunta problema apontada nesta revisão de escopo.

\section{Conclusão}

É incontestável que a Terapia nutricional adequada contribui para com a recuperação e a manutenção do estado nutricional do paciente oncológico. É esperado que o mapeamento de evidências científicas, com relação a fórmulas imunomoduladoras ofertadas no perioperatório de pacientes oncológicos em cirurgias gastrintestinal, contribua na prática clínica, proporcionando informações que possam aperfeiçoar o cuidado nutricional.

\section{Referências}

Aromataris, E. \& Munn, Z. (2020). JBI Manual for Evidence Synthesis. JBI. https://synthesismanual.jbi.global. https://doi.org/10.46658/JBIMES-20-01

Au, P. C. M., Li, H. L., Lee, G. K. Y., Li, G. H. Y., Chan, M., Cheung, B. M. Y., \& Cheung, C. L. (2021). Sarcopenia and mortality in cancer: A metaanalysis. Osteoporosis and sarcopenia, 7, S28-S33 https://doi.org/10.1016/j.afos.2021.03.002

Cardoso, B., Frazili, C., Liborio, F. S., Miranda, I., Neto, J., de Jesus, M. B., \& Freire, S. (2019). Impacto da terapia nutricional do pre-operatório de pacientes com câncer colorretal. Cadernos da Medicina-UNIFESO, 2(2). file:///C:/Users/gmsgo/Dropbox/My\%20PC\%20(LAPTOP-TUQOMC4J)/Downloads/1388$5475-1-\mathrm{PB} \% 20(3)$

de Oliveira, I. R. S, Andrade, G. D, \& de Souza, LP (2021). Desafios do fisioterapeuta nas repercussões disponíveis em pacientes acometidos pelo COVID-19: Protocolo de scoping review. Pesquisa, Sociedade e Desenvolvimento, 10 (7), e4910716032-e4910716032. https://doi.org/10.33448/rsd-v10i7.16032

Jordan, Z., Lockwood, C., Munn, Z. \& Aromataris, E. (2019). The updated Joanna Briggs Institute Model of Evidence-Based Healthcare. Int J Evid Based Healthc. 17(1):58-71. https://doi.org/10.1097/xeb.0000000000000155

Peters, M. D, Marnie, C., Tricco, A. C, Pollock, D., Munn, Z., Alexander, L., \& Khalil, H. (2020). Orientação metodológica atualizada para a realização de análises de escopo. Síntese de evidências JBI, 18 (10), 2119-2126. 10.11124 / JBIES-20-00167

Sierra, J. C, Dock-nascimento, D. B, Behne, T. E. G, Rodrigues, H. H. N. P, Andreo, F. O, Palauro, M. L, \& De-Aguilar-Nascimento, J. E (2020). Cirurgia oncológica de grande porte reduz a função muscular de pacientes com e sem risco nutricional. Revista do Colégio Brasileiro de Cirurgiões, 47. https://doi.org/10.1590/0100-6991e-20202470 
Research, Society and Development, v. 10, n. 16, e368101623599, 2021

(CC BY 4.0) | ISSN 2525-3409 | DOI: http://dx.doi.org/10.33448/rsd-v10i16.23599

Tiezerin, C. S., de Souza, D. H., Gonçalves, L. F., \& Haas, P. (2021). Impacto da Recusa Alimentar em Pacientes com Câncer: Revisão Integrativa da Literatura. Revista Brasileira de Cancerologia, 67(4). https://doi.org/10.32635/2176-9745.RBC.2021v67n4.1372

Tonon, A. P., \& Silva, P. S. T. (2020). Intervenções nutricionais na prevenção e tratamento de pacientes oncológicos em nível ambulatorial. International Journal of Nutrology, 13(03), 081-088. https://doi.org/10.1055 / s-0040-1718992

Tricco, A. C., Lillie, E., Zarin, W., O'Brien, K. K., Colquhoun, H., Levac, D., \& Straus, S. E. (2018). PRISMA extension for scoping reviews (PRISMA-ScR): checklist and explanation. Annals of internal medicine, 169(7), 467-473. https://doi.org/10.7326/M18-0850 